\title{
Relativistic calculation of the two-photon decay rate of highly-excited ionic states
}

\author{
Ulrich D. Jentschura ${ }^{1,2, *}$ and Andrey Surzhykov ${ }^{1,3}$ \\ ${ }^{1}$ Max-Planck-Institut für Kernphysik, \\ Postfach 103980, D-69029 Heidelberg, Germany \\ ${ }^{2}$ Institut für Theoretische Physik, Philosophenweg 16, 69120 Heidelberg \\ ${ }^{3}$ Physikalisches Institut der Universität, \\ Philosophenweg 12, 69120 Heidelberg
}

(Dated: November 20, 2018)

\begin{abstract}
Based on quantum electrodynamics, we reexamine the two-photon decay of one-electron atoms. Special attention is paid to the calculation of the (two-photon) total decay rates which can be viewed as the imaginary part of the two-loop self-energy. We argue that our approach can easily be applied to the cases with a virtual state having an intermediate energy between the initial and the final state of the decay process leading, thus, to the resonance peaks in the two-photon energy distribution. In order to illustrate our approach, we obtain fully relativistic results, resolved into electric and magnetic multipole components, for the two-photon decay rates of the $3 S_{1 / 2} \rightarrow 1 S_{1 / 2}$ transition in neutral hydrogen as well as in various hydrogen-like ions.
\end{abstract}

PACS numbers: 31.30.jn, 31.30.jc, 12.20.Ds, 32.80.Wr

*Electronic address: ulj@mpi-hd.mpg.de 


\section{INTRODUCTION}

Since the seminal work of Göppert-Mayer [1], two-photon decay rates of excited states in hydrogen-like atoms and ions have been the subject of intense experimental [2, 3, 4, 5] and theoretical [6, 7, 8, 9, 10, 11, 12, 13] studies. For many years, the investigations have dealt not only with the total decay rates but also with the energy and even angular distributions of the two emitted photons. By analyzing these (two-photon) properties, unique information has been obtained about the structural properties of one-electron systems including subtle relativistic effects as well as about the basic concepts of quantum physics such as, e.g., the entanglement.

Even though large experimental and theoretical efforts have been undertaken in the past to understand various aspects of the two-photon decay of hydrogen-like atoms, the analysis of this process still raises a number of unresolved problems. One of these problems, which currently attracts a lot of interest, concerns those two-photon transition from highly excited states to the ground state which pass through an intermediate state with a lower energy than the initial state of the two-photon process [9, 10] and can alternatively decay to the groundstate via two (or more) sequential one-photon emissions. Such a transition leads to resonance peaks in the energy spectrum of the coherently emitted photons from the two-photon decays which are located at the energies corresponding to the (real) intermediate states. One of the most pronounced examples of such a situation is the $3 S_{1 / 2} \rightarrow 1 S_{1 / 2}$ two-photon $E 1 E 1$ transition for which the differential (in energy) emission rate has singularities at energies corresponding to the $3 S_{1 / 2} \rightarrow 2 P_{1 / 2} \rightarrow 1 S_{1 / 2}$ and $3 S_{1 / 2} \rightarrow 2 P_{3 / 2} \rightarrow 1 S_{1 / 2}$ cascades. A proper treatment of these singularities is obviously required for computing total decay rates obtained after an integration over the energies of the coherently emitted photons in the direct two-photon decay $3 S_{1 / 2} \rightarrow 1 S_{1 / 2}$.

During the last two decades, the theoretical treatment of the resonances in the energy distribution of the emitted photons has been discussed in a number of places. In general, the decay rate $\Gamma_{i}$ of an initial state $|i\rangle$ in a hydrogenlike atom is the sum of a one-photon decay rate $\Gamma_{i}^{(1)}$ and a two-photon contribution $\Gamma_{i}^{(2)}$,

$$
\Gamma_{i}=\Gamma_{i}^{(1)}+\Gamma_{i}^{(2)}
$$

The expression for $\Gamma_{i}^{(2)}$ as originally derived in [1] is easily seen to involve an integral over the energies of the emitted photons, the sum of which has to be equal to the energy difference of 
the initial and final states, and a summation over all possible intermediate, virtual states. In order to avoid problems with non-integrable singularities, the authors of [9] have attributed $\Gamma_{i}^{(2)}$ only to the so-called non-resonant intermediate transitions, in contrast to a summation over the complete intermediate-state spectrum. The non-resonant transitions are given by intermediate states of energy higher than the energy $E_{i}$ of the initial state [9] (the "resonant" intermediate states, which are involved in the one-photon cascade processes, are explicitly excluded from the sum over intermediate states). Based on this assumption, the non-resonant contribution for the $3 S_{1 / 2} \rightarrow 1 S_{1 / 2}$ two-photon transition was calculated as $\Gamma_{3 S}^{(2)}=8.2196 s^{-1}$ for hydrogen. Later, this result has been also confirmed in the calculations by Florescu and co-workers [10] who used a different method for the summation over the "non-resonant" states.

Although the results presented in Refs. [9, 10] are in mutual agreement, they are both based on the interpretation of the two-photon decay rate $\Gamma_{i}^{(2)}$ as a rate generated only by nonresonant intermediate levels. In our manuscript, we would like to propose an alternative way for the computation of the (two-photon) total decay rates which leads to a natural removal of the infinities otherwise introduced into the expression for the two-photon decay rate. We apply here a fully relativistic, quantum electrodynamical approach to re-investigate the two-photon decay of highly-excited states of hydrogen-like atoms, paying special attention to a careful handling of the resonances infinitesimally displaced from Feynman's photon integration contour (these singularities exactly correspond to the problematic "resonant" intermediate states). By making use of this approach, we obtain finite, physically sensible results for the decay rates of the two-photon $3 S_{1 / 2} \rightarrow 1 S_{1 / 2}$ transitions in neutral hydrogen as well as in the various hydrogen-like ions. Apart of the leading, electric dipole $(E 1 E 1)$ transition, we also discuss the contributions from the higher multipole components to the total decay rate.

This paper is organized as follows: after a brief survey of the theoretical expressions used in our analysis (Sec. II), we proceed by discussing the method of evaluation (Sec. III) as well as the numerical results obtained for the differential as well as the total two-photon decay rates (Sec. [V]). Conclusions are given in Sec. V] 


\section{THEORY}

Within quantum electrodynamics, the (negative) imaginary part of the self-energy is just the $\Gamma / 2$, where $\Gamma$ is the decay width $[14,15,16]$. The one-photon decay $\Gamma^{(1)}$ rate is obtained from the imaginary part of the one-loop self-energy, while the two-loop self-energy gives rise to the two-photon decay rate $\Gamma^{(2)}$. Because the relativistic formulation of the two-loop selfenergy problem has been discussed before in a number of places [17, 18], we only mention here that by following a straightforward generalization of the standard procedure described for the non-relativistic framework in Refs. [12, 13, 16], we obtain the following expression for the two-photon decay rate $\left(\hbar=c=\epsilon_{0}=1\right)$,

$$
\Gamma_{i}^{(2)}=\frac{\alpha^{2}}{\pi} \lim _{\epsilon \rightarrow 0} \operatorname{Re} \int_{0}^{\omega_{\max }} \mathrm{d} \omega_{1} \omega_{1} \omega_{2} \int \mathrm{d} \Omega_{1} \mathrm{~d} \Omega_{2} S_{i f}\left(\omega_{1}, \omega_{2}\right),
$$

where $\omega_{1}+\omega_{2}=\omega_{\max }=E_{i}-E_{f}$ with the initial and final state energies $E_{i}$ and $E_{f}$, respectively. $S_{\text {if }}$ is given by

$$
\begin{gathered}
S_{i f}\left(\omega_{1}, \omega_{2}\right)=\sum_{\nu}\left(\frac{\left\langle\psi_{f}\left|\mathbf{A}_{1}^{*}\right| \psi_{\nu}\right\rangle\left\langle\psi_{\nu}\left|\mathbf{A}_{2}^{*}\right| \psi_{i}\right\rangle}{E_{i}-E_{\nu}-\omega_{2}+i \epsilon}\right. \\
\left.+\frac{\left\langle\psi_{f}\left|\mathbf{A}_{2}^{*}\right| \psi_{\nu}\right\rangle\left\langle\psi_{\nu}\left|\mathbf{A}_{1}^{*}\right| \psi_{i}\right\rangle}{E_{i}-E_{\nu}-\omega_{1}+i \epsilon}\right) \\
\times \sum_{\rho}\left(\frac{\left\langle\psi_{i}\left|\mathbf{A}_{1}\right| \psi_{\rho}\right\rangle\left\langle\psi_{\rho}\left|\mathbf{A}_{2}\right| \psi_{f}\right\rangle}{E_{i}-E_{\rho}-\omega_{1}+i \epsilon}\right. \\
\left.+\frac{\left\langle\psi_{i}\left|\mathbf{A}_{2}\right| \psi_{\rho}\right\rangle\left\langle\psi_{\rho}\left|\mathbf{A}_{1}\right| \psi_{f}\right\rangle}{E_{i}-E_{\rho}-\omega_{2}+i \epsilon}\right),
\end{gathered}
$$

where in the second factor, the initial and the final state are exchanged, but the infinitesimal imaginary part in the denominators remains $+i \epsilon$ (i.e., does not change sign). We here manifestly assume that $\psi_{i}(\boldsymbol{r}) \equiv \psi_{n_{i} j_{i} \mu_{i}}(\boldsymbol{r})$ and $\psi_{f}(\boldsymbol{r}) \equiv \psi_{n_{f} j_{f} \mu_{f}}(\boldsymbol{r})$ are the well-known solutions of the Dirac Hamiltonian for a single electron in the standard representation, describing an electron bound to a point-like nucleus with charge number $Z$. For photons propagating with wave vector $\boldsymbol{k}_{i}(i=1,2)$ and unit polarization vector $\boldsymbol{u}_{\lambda_{i}}\left(\boldsymbol{k}_{i} \cdot \boldsymbol{u}_{\lambda_{i}}=0\right)$, moreover, the electron-photon interaction operator $\mathbf{A}_{i}$ in the transition amplitude (3) can be written in velocity gauge as:

$$
\mathbf{A}_{i}=A_{0} \boldsymbol{\alpha} \cdot \boldsymbol{u}_{\lambda_{i}} \mathrm{e}^{i \boldsymbol{k}_{i} \boldsymbol{r}}
$$

where $A_{0}$ is a normalization factor, $\boldsymbol{\alpha}$ are the standard Dirac matrices, and $\lambda_{i}= \pm 1$ denotes the helicity, i.e. the spin projection of the photon onto the direction of propagation $\boldsymbol{k}_{i}$. It is 
important to note that even though the electron-photon interaction operator (4) depends, of course, on the direction of the photon emission, one has to integrate over these directions in Eq. (2) in order to get the total decay rate.

\section{EVALUATION}

The summation over the intermediate states in the amplitude (3) runs over the complete one-particle spectrum $\left|\psi_{\nu}\right\rangle \equiv\left|\psi_{n_{\nu} j_{\nu} \mu_{\nu}}\right\rangle$, including a summation over the discrete part of the spectrum as well as an integration over the positive and negative-energy continuum of the Dirac spectrum. One has to use the full Dirac-Coulomb Green function-which is not known in closed analytic form - in order to perform this calculation consistently. In recent years, the Green's function method [19] has been widely applied for the analysis of the total two-photon decay rates as well as the photon-photon angular correlation functions [20]. Various possibilities for the numerical implementation of the relativistic Green's function are known, among which we would like to mentionas (i) a well-known formulation in terms of Whittaker functions [21] and (ii) a Sturmian decomposition in terms of Laguerre polynomials as suggested by Hylton and Snyderman [22].

We use the latter representation and apply the techniques of Racah's algebra to all spherical tensors and to the standard radial-angular representation of the wave functions, and to the Dirac-Coulomb Green function. For the interaction of electrons with the radiation field, the spherical tensor components are obtained from the known standard multipole expansion of the photon operator [see, e.g., Eq. (5) of Ref. [23]],

$$
\boldsymbol{u}_{\lambda} \mathrm{e}^{i \boldsymbol{k r}}=\sqrt{2 \pi} \sum_{L M p} i^{L}(i \lambda)^{p} \sqrt{2 L+1} \boldsymbol{A}_{L M}^{(p)} D_{M \lambda}^{L}(\boldsymbol{n})
$$

where $\boldsymbol{A}_{L M}^{(p)}$ denotes the electric $(p=1)$ and magnetic $(p=0)$ multipole fields, respectively.

\section{RESULTS}

The great advantage of the multipole decomposition (5) is that is allows us to study the contributions to the total (two-photon) decay rate from the various allowed multipole combinations. We use the integrand in the integral over $\omega_{1}$ in Eq. (3) as a measure of the differential decay rate (where we can set explicitly $\epsilon=0$ for the differential rate). 

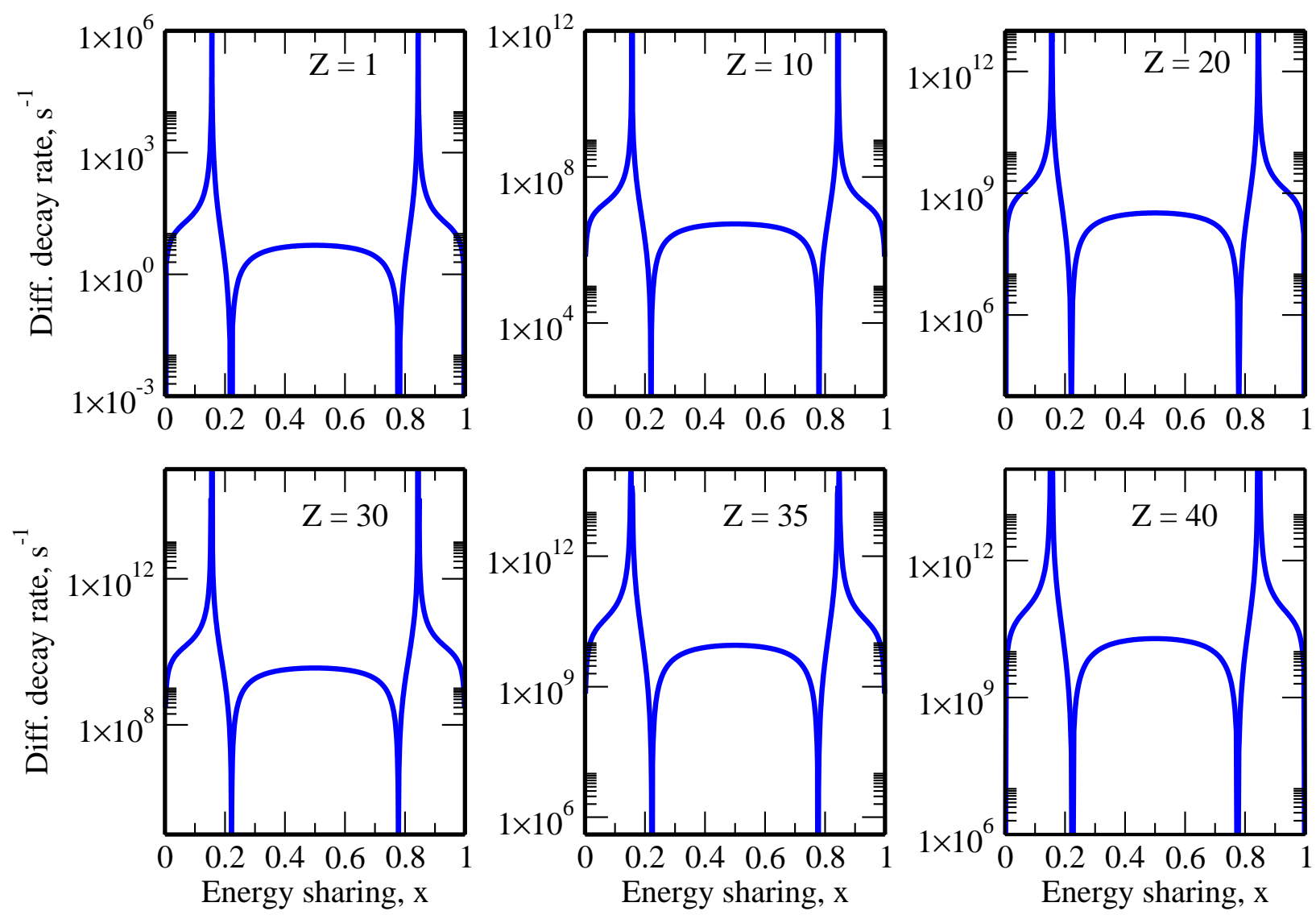

FIG. 1: (Color online) Differential decay rate $\mathrm{d} \Gamma / \mathrm{d} x$ for the dominant E1E1 component of the $3 S \rightarrow 1 S$ two-photon transitions in neutral hydrogen as well as in hydrogen-like ions with $Z=$ $1,10,20,30,35$ and 40 , where $x$ is the energy sharing $x=\omega_{1} /\left(\omega_{1}+\omega_{2}\right)$. Relativistic wave functions are used for the initial, intermediate and final states, but the electron-photon interaction has been restricted to electric dipole term ( $E 1 E 1$ term). The resonance peaks in the decay rate correspond to the resonant $3 S_{1 / 2} \rightarrow 2 P_{1 / 2} \rightarrow 1 S_{1 / 2}$ and $3 S_{1 / 2} \rightarrow 2 P_{3 / 2} \rightarrow 1 S_{1 / 2}$ decay processes.

The energy distributions of the two photons emitted in the $3 S_{1 / 2} \rightarrow 1 S_{1 / 2}$ decay of neutral hydrogen and hydrogen-like ions are calculated as a function of the energy sharing parameter $x=\omega_{1} /\left(\omega_{1}+\omega_{2}\right)$. For an energy sharing in the range $0<x<1$, the contributions to the energy distribution from the $E 1 E 1$ and $E 1 M 2$ multipole combinations are displayed in Figs. 1 and 2, respectively. As seen from these figures, the photon energy distributions for both multipole combinations exhibit sharp resonance peaks. As already mentioned, this behaviour is due to the fact that the summation in Eq. (3) includes also intermediate states $|\nu\rangle$ having an energy $E_{\nu}$ with $E_{i}>E_{\nu}>E_{f}$. However, the intermediate states contributing 

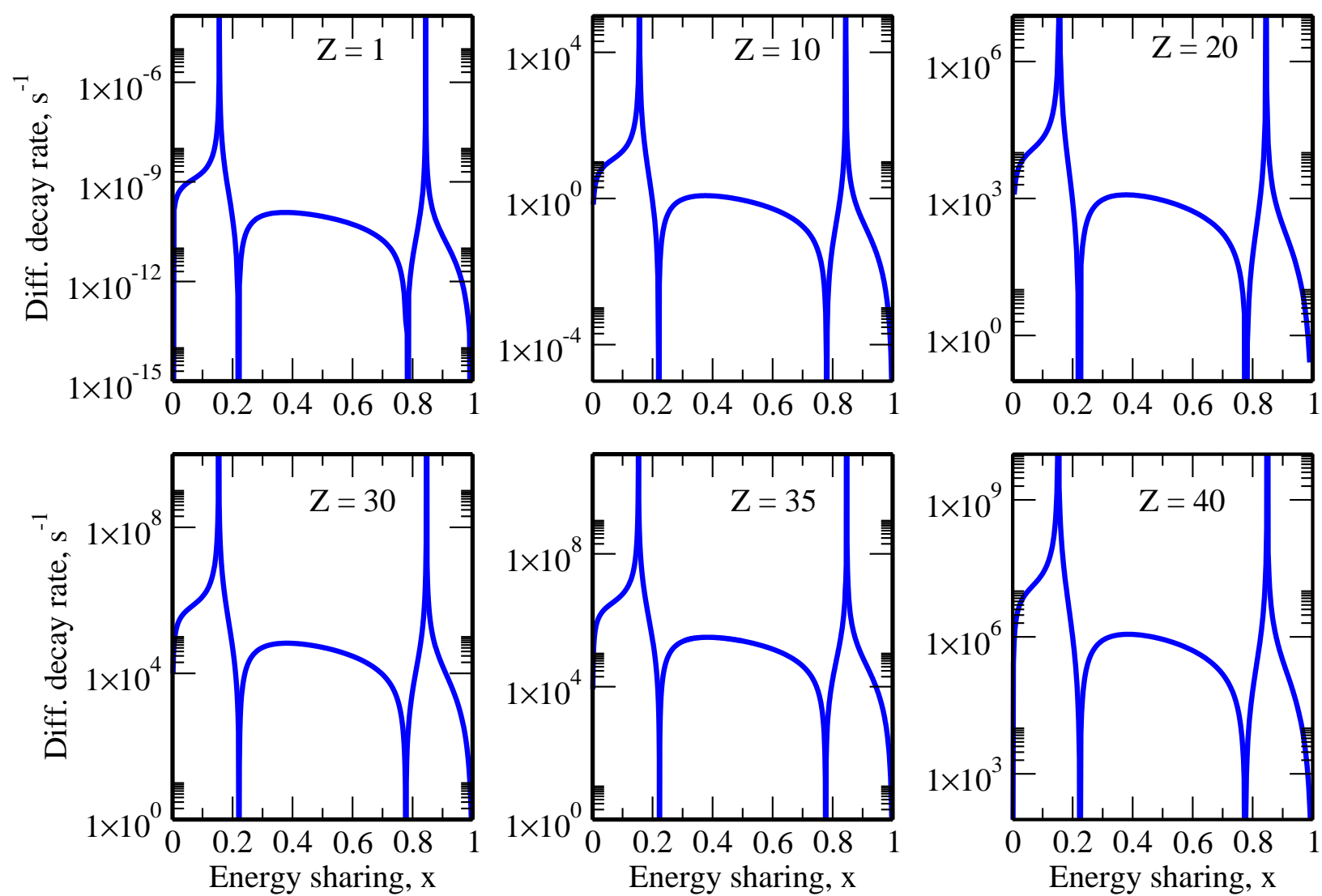

FIG. 2: (Color online) Differential decay rate $\mathrm{d} \Gamma / \mathrm{d} x$ for the E1M2 component of the $3 S \rightarrow 1 S$ twophoton transitions in neutral hydrogen as well as in hydrogen-like ions with $Z=1,10,20,30,35$ and 40 ( $x$ is the fraction of energy carried by the E1 photon). The E1M2 term is treated relativistically. The resonances in the decay rate exclusively correspond to the $3 S_{1 / 2} \rightarrow 2 P_{3 / 2} \rightarrow 1 S_{1 / 2}$ cascade, the $2 P_{1 / 2}$ state does not contribute.

to the peaks are not only defined by the (symmetry of) the initial $|i\rangle$ and final $|f\rangle$ states but also by the multipole components of the radiation field involved in the two-photon process and are different for $E 1 E 1$ as opposed to $E 1 M 2$, and in addition, marked differences exist between the low- $Z$ and the high- $Z$ region. E.g., the fine-structure of the resonance in the $E 1 E 1$ energy spectrum grows with $Z$ (the contributing states are $2 P_{1 / 2}$ and $2 P_{3 / 2}$ ). By contrast, no splitting is observed - even for very heavy ions - for the E1M2 component of the $3 S_{1 / 2} \rightarrow 1 S_{1 / 2}$ decay. Only one intermediate state, namely $2 P_{3 / 2}$, is allowed for $E 1 M 2$.

Our treatment of the intermediate state resonance peaks in the integration over the photon energy is dictated by an accurate analysis of Eqs. (2) -(3) as obtained form the relativistic two-loop self energy. The general structure of the contribution to $S_{i f}\left(\omega_{1}, \omega_{2}\right)$ 
generated by virtual states with intermediate energies $E_{i}>E_{\nu}>E_{f}$ with resonance energy $\omega_{r}=E_{i}-E_{\nu}$ or $\omega_{r}=E_{\nu}-E_{f}$ is as follows:

$$
S_{i f}\left(\omega_{1}, \omega_{2}\right) \sim \frac{R_{1}}{\omega_{r}-\omega+i \epsilon}+\frac{R_{2}}{\left(\omega_{r}-\omega+i \epsilon\right)^{2}} .
$$

The integration of the first term can be carried out using the Dirac prescription (see, for example, Ref. [12]). The second term of Eq. (66) can be treated using the formula

$$
\lim _{\epsilon \rightarrow 0} \operatorname{Re} \int_{0}^{1} \mathrm{~d} \omega\left(\frac{1}{\omega_{r}-\omega+i \epsilon}\right)^{2}=\frac{1}{\omega_{r}\left(\omega_{r}-1\right)}
$$

where we used an appropriate scaling of the photon energy integration variable in order to map the integration region to the interval $(0,1)$. It is important to note that Eq. (7) holds strictly for $0<\omega_{r}<1$, but the limit is not approached uniformly [12, 13]; i.e., it would be forbidden to exchange the sequence of the limit $\epsilon \rightarrow 0$ with the integration over $\omega$. As usual in quantum electrodynamic processes, all regulators have to be kept up until the very end of the calculation.

With these preparations, it is easy now to integrate over the photon energies (see Table 1 for the $3 S_{1 / 2} \rightarrow 1 S_{1 / 2}$ process). As seen from the table, the cross sections for the $E 1 E 1$, $E 1 M 2$ and $M 1 M 1$ components components scale with the nuclear charge as $Z^{6}, Z^{10}$ and $Z^{10}$, respectively. As expected, this scaling behaviour is similar to the $Z$-scaling of the multipole components in the $2 S_{1 / 2} \rightarrow 1 S_{1 / 2}$ transition.

Furthermore, as seen from Table 1 and as implied by the non-uniform convergence of the integrals, the intermediate states with the energies $E_{\nu}$ lying between the energies of the initial and the final states give a finite contribution to the two-photon decay rate. For the electric dipole $(E 1 E 1)$ transition in a neutral hydrogen atom, e.g., a proper treatment of the intermediate $2 P_{1 / 2}$ and $2 P_{3 / 2}$ states leads to the decay rate of $\Gamma_{3 S}^{(2)}=2.08 s^{-1}$ which is in agreement with the result of nonrelativistic calculations reported in Ref. [12]. However, when comparing our prediction with the theoretical data by Cresser and co-workers [9] a large discrepancy by about a factor of 4 is observed. The occurrence of the discrepancy is natural because the problematic virtual states with intermediate energies are treated differently in [9]. 


\begin{tabular}{cccc}
\hline \hline$Z$ & $\begin{array}{c}E 1 E 1 \\
\left(\mathrm{Z}^{-6}\right)\end{array}$ & $\begin{array}{c}E 1 M 2 \\
\left(\mathrm{Z}^{-10} 10^{10}\right)\end{array}$ & $\begin{array}{c}M 1 M 1 \\
\left(\mathrm{Z}^{-10} 10^{12}\right)\end{array}$ \\
\hline 1 & 2.08 & 1.19 & 6.13 \\
5 & 2.03 & 1.18 & 6.13 \\
10 & 1.98 & 1.16 & 6.14 \\
15 & 1.94 & 1.14 & 6.16 \\
20 & 1.90 & 1.12 & 6.20 \\
25 & 1.84 & 1.08 & 6.24 \\
30 & 1.79 & 1.03 & 6.30 \\
35 & 1.67 & 0.96 & 6.39 \\
40 & 1.60 & 0.86 & 6.50 \\
\hline \hline
\end{tabular}

TABLE I: Contributions from different combinations of multipoles to the integrated decay rate $\Gamma^{(2)}$, in units of radians per second. Relativistic calculations have been performed for different hydrogen-like ions.

\section{CONCLUSIONS}

The two-photon decay of hydrogen-like ions has been re-investigated within the framework of relativistic quantum electrodynamics. Starting from first principles of this theory, we treat the total (two-photon) decay rate as the imaginary part of the relativistic two-loop self-energy. The great advantage of this approach, which has its roots in field theory, is that it provides a simple and efficient route to handle the potentially problematic cases of those two-photon transitions from an excited into the ground state which pass intermediate states that can otherwise also be reached in one-photon cascades from the initial to the final states. We found that those states with energies that lie between the energy of the initial and the final states, contribute a finite correction to the total two-photon decay rate. Taking into account this correction, we calculate the rates for the $3 S_{1 / 2} \rightarrow 1 S_{1 / 2}$ two-photon decay of neutral hydrogen as well as hydrogen-like ions. Our results are in a good agreement with nonrelativistic calculations for low $Z$ (see Refs. [12, 13]) but show a significant deviation from the data by Cresser and co-workers [9]. 
Our quantum electrodynamics approach, as discussed in the present paper, opens a way for a systematic theoretical analysis of the simultaneous, coherent two-photon emission from one-electron (and many-electron) atomic systems, even in cases where problematic intermediate states with an energy between the initial and final states give rise to resonance peaks in the photon energy distributions. We stress here that a conceivable alternative approach to the removal of the formal infinities generated by the intermediate "cascade" states, which is based on the explicit removal of these states from the sums over $\nu$ and $\rho$ in Eq. (2), gives rise to a number of conceptual problems, including gauge-noninvariance with respect to length and velocity gauges [13]. Our approach is manifestly gauge invariant and also avoids problems connected with the identification of the infinitesimal parts $i \epsilon$ in the propagator denominators in Eq. (2) with partial or total decay rates of the intermediate states: the $\epsilon$ parameters are free parameters which approach zero after all other operations, including the integrations over the photon energies, have been performed. This operation leads to a finite result and corresponds, as explained in Ref. [13], to a partial removal of the problematic intermediate states from the sum over all virtual states involved in the two-photon process, albeit in a fully gauge-invariant manner.

In addition to its relevance for atomic physics, our approach may have a significant impact for astrophysical studies where a detailed knowledge of the (properties of) two-photon transitions is highly required for the analysis of cosmological hydrogen and helium recombination. The contribution of two-photon processes to the recombination history represents an issue which has recently attracted substantial theoretical interest [24].

\section{ACKNOWLEDGMENTS}

U.D.J. acknowledges support from Deutsche Forschungsgemeinschaft (Heisenberg program), and A.S. acknowledges support from the Helmholtz Gemeinschaft (Nachwuchsgruppe VH-NG-421). The authors acknowledge insightful discussions with Z. Harman regarding

the Sturmian decomposition of the Dirac-Coulomb Green function as given in Ref. [22], and helpful discussions with P. Indelicato on general aspects of the two-photon decay and 
associated resonances.

[1] M. Göppert-Mayer, Ann. Phys. (Leipzig) 401, 273 (1931).

[2] M. Lipeles, R. Novic, and N. Tolk, Phys. Rev. Lett. 15, 690 (1965).

[3] R. Marrus and R. W. Schmieder, Phys. Rev. A 5, 1160 (1972).

[4] W. Perrie, A. J. Duncan, H. J. Beyer, and H. Kleinpoppen, Phys. Rev. Lett. 54, 1790 (1985).

[5] P. H. Mokler and R. W. Dunford, Phys. Scr. 69, C1 (2004).

[6] G. Breit and E. Teller, Astrophys. J. 91, 215 (1940).

[7] G. W. F. Drake and S. P. Goldman, Phys. Rev. A 23, 2093 (1981).

[8] V. Florescu, Phys. Rev. A 30, 2441 (1984); J. H. Tung, X. M. Ye, G. J. Salamo, and F. T. Chan, Phys. Rev. A 30, 1175 (1984); V. Florescu, S. Patrascu, and O. Stoican, Phys. Rev. A 36, 2155 (1987).

[9] J. D. Cresser, A. Z. Tang, G. J. Salamo, and F. T. Chan, Phys. Rev. A 33, 1677 (1986).

[10] V. Florescu, I. Schneider, and I. N. Mihailescu, Phys. Rev. A 38, 2189 (1988).

[11] J. P. Santos, F. Parente, and P. Indelicato, Eur. Phys. J. D 3, 43 (1998).

[12] U. D. Jentschura, J. Phys. A 40, F223 (2007).

[13] U. D. Jentschura, J. Phys. A 41, 155307 (2008).

[14] R. Barbieri and J. Sucher, Nucl. Phys. B 134, 155 (1978).

[15] A. O. Barut and Y. I. Salamin, Phys. Rev. A 43, 2524 (1991).

[16] U. D. Jentschura, Phys. Rev. A 69, 052118 (2004).

[17] J. A. Fox and D. R. Yennie, Ann. Phys. 81, 438 (1973).

[18] V. A. Yerokhin, P. Indelicato, and V. M. Shabaev, Eur. Phys. J. D 25, 203 (2003).

[19] R. A. Swainson and G. W. F. Drake, J. Phys. A 24, 95 (1991).

[20] A. Surzhykov, P. Koval, and S. Fritzsche, Phys. Rev A 71, 022509 (2005).

[21] P. J. Mohr, Ann. Phys. (N.Y.) 88, 26 (1974); Ann. Phys. (N.Y.) 88, 52 (1974).

[22] D. J. Hylton and N. J. Snyderman, Phys. Rev. A 55, 2651 (1997).

[23] J. Eichler, A. Ichihara, and T. Shirai, Phys. Rev. A 58, 2128 (1998).

[24] V. K. Dubrovich and S. I. Grachev, Astron. Lett. 31, 359 (2005); W. Y. Wong and D. Scott, Mon. Not. R. Astron. Soc. 375, 1441 (2007); J. Chluba and R. A. Sunyaev, e-print 0705.3033 [astro-ph]. 